\title{
JUURNAL.RU
}

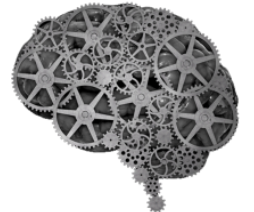

COMPANY GROUP "INTELLEKT"

Топоров P.B. Вятский государственный университет

Киров, Россия

doi: 10.18411/1j2016-4-46

\section{Применение метода проблемной лекции в вузе на примере изучения темы «комплексные числа»}

Одной из важнейших форм обучения в вузе является лекция. Вузовская лекция - главное звено дидактического цикла обучения. Ее цель - формирование ориентировочной основы для последующего усвоения студентами учебного материала.

Существуют различные виды лекций. В данной работе мы более подробно рассмотрим один из них - проблемную лекцию.

Проблемная лекция чаще всего начинается с вопроса, с постановки проблемы, а дальнейшее изложение учебного материала подается как решение обозначенной проблемы. Чтение проблемных лекций дает возможность достижения важнейших целей организации обучения:

- повышение мотивации и внедрение «технологии» поиска;

- обеспечение самостоятельного переосмысления и усвоения новых теоретических знаний студентами;

- развитие теоретического мышления будущего учителя

На проблемной лекции происходит «открытие» самими студентами новых знаний. Она строится таким образом, что деятельность студентов приближается к поисковой, при которой задействованы и мышление студента, и его личностное отношение к усваиваемому материалу. На традиционной лекции студенты 
получают необходимую информацию (знания, алгоритм решения и др.), а затем следуют примеры применения этих знаний. На проблемной же лекции преподаватель создает проблемную ситуацию, а студенты самостоятельно пытаются найти пути ее разрешения еще до того, как они получат новые для них знания.

Лекция становится проблемной при выполнении двух условий.

1. Содержание учебного материала отобрано и структурировано с учетом принципа проблемности (преподавателем разработана система учебных проблемных задач, отражающих основное содержание лекции).

2. Принцип проблемности реализован при развертывании этого содержания непосредственно на лекции (лекция построена как диалогическое общение преподавателя со студентами в ходе разрешения поставленных проблем).

Далее рассмотрим пример применения метода проблемной лекции в начале изучения студентами темы «комплексные числа».

В начале лекции говорится о том, что системы действительных чисел недостаточно для того, чтобы решать любые квадратные уравнение с действительными коэффициентами, в частности квадратные уравнения с отрицательным дискриминантом.

Говорится, что цель лекции в том, чтобы расширить множество $\boldsymbol{R}$ до такой системы чисел, в которой каждое квадратное уравнение имеет корни. Это и есть проблемная задача. При этом новое числовое множество (обозначим его $\boldsymbol{C}$ ) должно удовлетворять следующим условиям:

1) Любое квадратное уравнение должно быть разрешимо в нём.

2) Оно должно содержать в себе множество $\boldsymbol{R}$ в качестве подмножества.

3) Нужно определить операции сложения и умножения в $\boldsymbol{C}$ таким образом, чтобы оно являлось полем, при этом $0 \in \boldsymbol{R}$ должен быть нейтральным элементом по сложению в $\boldsymbol{C}$, a $1 \in \boldsymbol{R}$ нейтральным элементом по умножению в C . 
4) Полученное множество должно иметь хорошую геометрическую интерпретацию. (Для $\boldsymbol{R}$ геометрической интерпретацией является числовая прямая.)

Вначале рассматривается простейшее квадратное уравнение $x^{2}+1=0$ или $x^{2}=-1$. Даже оно не имеет действительных корней. Его решение обозначается $i$ и называется мнимой единицей, т.е. $i^{2}=-1, i=\sqrt{-1}$. Далее рассматриваются уравнения вида $x^{2}=-a$, где $a>0$, соответственно, $-a<0$. В ходе обсуждения устанавливается, что решение таких уравнений имее вид $x=\sqrt{-a}=\sqrt{a \cdot(-1)}=\sqrt{a} \cdot \sqrt{-1}=\sqrt{a} i$. Таким образом, мы можем получить значения квадратного корня из любого отрицательного числа: $\sqrt{-4}=2 i, \sqrt{-10}=\sqrt{10} i$ и т.д. Если $i$ мнимая единица, то числа вида $b i$, где $b \in \boldsymbol{R}$ называются чисто мнимыми. Далее рассматривается квадратное уравнение произвольного вида с отрицательным дискриминантом. Например, $k x^{2}+l x+m=0, \quad$ причём $D=l^{2}-4 k m<0$. Тогда его корни будут иметь вид $x_{1,2}=\frac{-l \pm \sqrt{D}}{2 k}=\frac{-l \pm \sqrt{|D|} i}{2 k}=-\frac{l}{2 k} \pm \frac{\sqrt{|D|}}{2 k} i$. Получается, что корень произвольного квадратного уравнения с отрицательным дискриминантом является суммой действительного числа и чисто мнимого числа. Далее можно определить множество $\boldsymbol{C}$ комплексных чисел. Числа вида $a+b i$, где $a, b \in \boldsymbol{R}$, a $i$ - мнимая единица, называется комплексными.

Дальше выясняется, что $\boldsymbol{R}$ содержится в $\boldsymbol{C}$ в качестве подмножества. Для этого рассмотрим произвольное комплексное число $z=a+b i$. Пусть $b=0$, откуда следует, что $z=a+0 i=a \in R$. То есть множество действительных чисел является подмножеством комплексных чисел.

Далее перед студентами ставится проблема введения на $\boldsymbol{C}$ операций сложения и умножения так, чтобы оно являлось полем.

a) Сложение. Даны комплексные числа $z_{1}=a+b i$ и $z_{2}=c+d i$ $(a, b, c, d \in \boldsymbol{R})$. Самым простым и очевидным способом является следующий спо- 
соб нахождения суммы: $z_{1}+z_{2}=(a+c)+(b+d) i$. Остаётся только проверить, чтобы выполнялись все аксиомы поля, связанные со сложением, а именно:

1) коммутативность сложения,

2) ассоциативность сложения,

3) наличие нейтрального элемента по сложению $(0=0+0 i)$,

4) наличие противоположного элемента по сложению. (Для $a+b i$ противоположным будет $-a-b i)$

Все аксиомы легко проверяются напрямую. В итоге делается вывод, что такое определение сложения комплексных чисел подходит для того, чтобы $\boldsymbol{C}$ было полем.

б) Умножение. Даны комплексные числа $z_{1}=a+b i$ и $z_{2}=c+d i$ $(a, b, c, d \in \boldsymbol{R})$. Студентами может быть сделано предположение, что умножение комплексных чисел можно определить по аналогии со сложением, то есть $z_{1} z_{2}=a c+b d i$. Чтобы обосновать неверность данного предположения можно привести следующий пример. Пусть $z_{1}=a+b i$, а $z_{2}=1=1+0 i$. Так как $1-$ нейтральный элемент по умножению, то должно быть верно равенство $z_{1} z_{2}=z_{1}$. Но по нашему определению умножения получаем, что $z_{1} z_{2}=a+0 i=a \neq z_{1}$.

Если рассмотреть комплексное число как сумму двух слагаемых, то произведение двух комплексных чисел можно представить как произведение двух скобок, которые для получения результата нужно раскрыть, то есть $z_{1} z_{2}=(a+b i)(c+d i)=a c+b c i+a d i+b d i^{2}=a c+b c i+a d i-b d=$ $=(a c-b d)+(b c+a d) i$

Если умножение определить таким способом, то все аксиомы поля для умножения также будут выполняться.

1) коммутативность умножения,

2) ассоциативность умножения,

3) наличие нейтрального элемента по умножению $(1=1+0 i)$, 
4) наличие обратного элемента для любого ненулевого (для $z=a+b i \neq 0$ обратным будет $z^{-1}=\frac{a}{a^{2}+b^{2}}-\frac{b}{a^{2}+b^{2}} i$ ).

Также легко будет проверить, что умножение дистрибутивно относительно сложения. Таким образом, $\boldsymbol{C}$ с данными операциями сложения и умножения действительно является полем.

Можно вспомнить, что геометрической интерпретацией поля действительных чисел является числовая прямая. То есть каждому действительному числу соответствует точка на числовой прямой и каждой точке числовой прямой соответствует действительное число. Так как произвольное комплексное число имеет вид $a+b i$, то логично поставить ему в соответствие точку $M(a, b)$ плоскости. Опять же несложно заметить, что каждому комплексному числу соответствует точка на плоскости и каждой точке плоскости соответствует комплексное число.

Вывод. В данной работе рассмотрено применение метода проблемной лекции при изучении темы «комплексные числа». Проблемные лекции способствуют творческому усвоению студентами закономерностей, принципов и содержания изучаемой науки, активизируют их самостоятельную (аудиторную и внеаудиторную) учебно-познавательную деятельность. 


\section{Литература:}

1. Матюшкин А.М. Проблемы развития профессионально-теоретического мышления. - М.: Педагогика, 1980.

2. Вербицкий А.А. Активное обучение в высшей школе: контекстный подход. М.: Высшая школа, 1991.

3. Курош А.Г. Курс высшей алгебры. - М.: Наука, 1968. 